\title{
RECUPERANDO UN PROGRAMA KUHNIANO EN HISTORIA DE LA CIENCIA
}

\author{
RECOVERING A KUHNIAN PROGRAM \\ IN HISTORY OF SCIENC
}

Leandro Giri* y Matías Giri*

\begin{abstract}
Resumen
En el presente trabajo analizaremos la propuesta de programa historiográfico de Thomas Kuhn. Para ello, mostraremos que La estructura de las revoluciones científicas, siendo la obra más leída y comentada del autor, presentó falencias que llevaron a grandes esfuerzos de refinamiento posterior. Este refinamiento se dio en el resto de la obra de Kuhn, pero quedó registrado mayormente de manera asistemática. Sin embargo, mostraremos que el inédito y recientemente recuperado y traducido texto del autor Desarrollo científico y cambio de léxico constituye una fuente primaria sintética y sistemática que resume su programa en su versión más refinada, tras reforzar su posición debido a las críticas suscitadas por La estructura.
\end{abstract}

Palabras clave: Thomas Kuhn, Historia de la Ciencia, Conferencias Thalheimer, Anti-presentismo, Internalismo kuhniano.

\begin{abstract}
In this work we will analyze Thomas Kuhn's proposal of historiographic program. We will show that The Structure of Scientific Revolutions, being the most read and commented work of the author, presented failures because of which great efforts of posterior refinement were needed. This refining job was done in the rest of Kuhn's work, but it was registered mostly asystematically. Howe-
\end{abstract}

* L. Giri. Doctor en Epistemología e Historia de la Ciencia, IIF-Sociedad Argentina de Análisis Filosófico / CONICET, Argentina. E-Mail: leandrogiri@gmail.com

* M. Giri. Profesor de la Universidad de Buenos Aires, Argentina. E-Mail: matiasgiri@outlook.com 
ver, we will show that the previously unpublished and recently recovered and translated to Spanish text Scientific Development and Lexical Change is a synthetic and systematic primary source that summarizes his program in its most refined version, after reinforcing his position against the critics done to The Structure.

Keywords: Thomas Kuhn, History of Science, Thalheimer Conferences, Anti-presentism, Kuhnian Internalism.

Recibido: 2019-06-29 Aceptado: 2020-04-20

\section{Introducción}

Cuando se hace referencia a Thomas Kuhn, lo primero que aparece en el radar es su trabajo más leído: La estructura de las revoluciones científicas (197I [1962]), en adelante ERC. A pesar del éxito logrado por ERC, este ha sido objeto de múltiples críticas que llevaron a Kuhn a dedicar grandes esfuerzos a refinar su posición filosófica e historiográfica.

La intención de nuestro análisis no es la de restar valor a ERC, sino la de advertir sobre el valor de la obra posterior, en particular para quienes deseen acercarse a la historia de la ciencia. Resulta que este es un momento excelente para volver a las ideas kuhnianas, ya que se ha publicado el texto inédito, en idioma español, de las Conferencias Thalheimer dictadas por el autor en la Universidad Johns Hopkins en 1984, bajo el nombre Desarrollo científico y cambio de léxico, en adelante DCCL (Kuhn, 20I7). Este texto posee la ventaja de condensar en un formato relativamente breve las ideas kuhnianas post-ERC con un lenguaje accesible. Así, DCCL aparece como una nueva fuente primaria que desarrolla el marco kuhniano de una manera que resulta al mismo tiempo sistemática, rigurosa y pedagógica, y mucho más actualizada que su best seller. Parece atendible la sugerencia de que aquellos docentes que en este momento utilicen ERC como material para sus clases acerca de Kuhn se planteen acercarse a DCCL. Este además posee un valiosísimo estudio introductorio por parte del filósofo Pablo Melogno, donde se analiza en detalle la relación entre las Conferencias Thalheimer y el resto de la obra kuhniana publicada, aportando un criterio de ordenamiento de enorme utilidad para delinear la evolución de las ideas del autor.

El presente trabajo se organizará de la siguiente manera. En primer lugar, se analizará la importancia de la filosofía historicista de la ciencia (o la historia de la ciencia mediante reconstrucciones racionales), tradición 
académica iniciada en 1962 con ERC. En segundo lugar se reconstruirá brevemente el programa en historia de la ciencia que presenta Kuhn en ERC enfatizando las debilidades de tal programa, que lo llevaron a realizar múltiples modificaciones a su propuesta, modificaciones presentes de forma dispersa y asistemática en su obra posterior. Por último, mostraremos que dichas problemáticas son atendidas en DCCL, con lo que éste constituye un buen compilado de las ideas del autor en su etapa madura.

Antes de proceder con el cuerpo principal del trabajo, nos permitimos una digresión para referirnos a la importancia de ERC en la filosofía e historia de la ciencia contemporánea.

ERC resultó disruptivo en el momento de su publicación, puesto que introdujo muchas novedades a la filosofía de la ciencia en un formato simple y didáctico. A través de esta obra el autor alcanzó el pináculo del éxito académico, siendo dicho texto el más vendido en el área de la filosofía de la ciencia de todos los tiempos.

La huella de ERC ha sido profunda en la historia intelectual del Siglo XX, y continúa siéndolo hoy, bien entrados en el Siglo XXI. Ian Hacking (2012) afirma que hacia 1987 había vendido casi 650.000 copias y aparecido en múltiples listas de "los mejores libros del Siglo", como así también de los más citados.

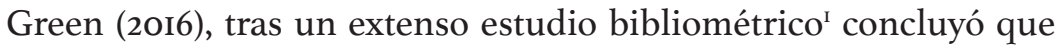
ERC, a más de medio siglo de su publicación, era el libro más citado de las ciencias sociales. También afirmó que la relación de citas entre ERC y la segunda obra más citada de Kuhn es algo mayor a 20, lo que significa que su best seller ha sido al menos 20 veces más citado que cualquiera de sus textos posteriores (o anteriores). La aseveración puede ser matizada al analizar que gran parte de las referencias a ERC son de carácter protocolar: cada mención al término "paradigma" que hay en la literatura suele venir acompañada de una referencia a Kuhn, aunque muchas veces no se trate de menciones legítimamente interesadas en la propuesta del autor. Aún así, el impacto de ERC es incuestionable.

La ubicuidad e importancia de ERC se trasunta en los planes de estudio de casi toda asignatura universitaria dedicada a la filosofía de la ciencia, siendo dicha asignatura, a su vez, horizontal a muchos programas curriculares de universidades a nivel mundial.

I El estudio de Green cubre a la antropología, economía, educación, geografía, lingüística, management, filosofía, ciencias políticas y psicología, lo cual no incluye a las ciencias naturales y tampoco a la historia, disciplinas sobre las que Kuhn también influyó fuertemente. 
¿Por qué es ERC tan importante entonces en la historia intelectual contemporánea? La pregunta no es trivial, puesto que múltiples voces críticas desde la filosofía de la ciencia entendieron a Kuhn como un defensor de una ciencia irracional, o al menos un escritor demasiado poco riguroso para los estándares del mainstream de la filosofía anglosajona, más acostumbrados a los métodos formales de análisis de las teorías científicas (p.e. Popper 1970; Watkins 1970; Shapere 1964; Sheffler 1967).

Sin dudas, ERC cumplió en su momento un rol rupturista. Según Hacking (2012) los elementos novedosos incluían: la mejora de la teoría de la contrastación falsacionista de Popper, la introducción de términos novedosos para la filosofía de la ciencia, la apertura a los estudios sociológicos para complementar los análisis sobre la dinámica científica y el desafío a los cánones establecidos por la filosofía tradicional acerca del progreso de la ciencia. A pesar de que filósofos como Bar-Am (20I4) afirmen que tales elementos no fueron realmente innovadores sino que ya se encontraban en la filosofía pre-ERC, ciertamente es Kuhn quien los coloca definitivamente en la agenda del mainstream.

Además debería agregarse la propuesta de una imbricación profunda entre dos disciplinas de segundo orden: la filosofía de la ciencia por un lado, y la historia de la ciencia por el otro. Dicha imbricación llevó a que surgiera un movimiento dentro de la filosofía de la ciencia llamado "giro historicista", "filosofía historicista de la ciencia" o "nueva filosofía de la ciencia". La idea era diferenciar a aquellos que construían su concepción epistemológica a partir del análisis de la historia de la ciencia (y a su vez construían la historia de la ciencia a partir de una lente epistemológica) de aquellos que pertenecían a la concepción tradicional (en general seguidores del positivismo lógico o del racionalismo crítico popperiano, más adeptos a los métodos formalistas de análisis de teorías científicas). Entre los autores más reconocidos dentro de la mencionada corriente historicista, además del propio Kuhn, se encontraban grandes filósofos como Imre Lakatos, Paul Feyerabend, Karl Polanyi y Mary Hesse, entre otros.

Muchos epistemólogos profesionales se dedicaron a trabajar en historia de la ciencia entre la década del 60' y los ' 80 (momento en que el historicismo fue paulatinamente reemplazado por otros movimientos en el mainstream de la filosofía de la ciencia), ya sea para construir mejores marcos epistemológicos o por mero interés historiográfico, o por las dos cosas. Los marcos epistemológicos se constituyeron entonces como "recetas" metodológicas para construir relatos de historia de la ciencia (ver Lakatos, 1987). En estos esquemas, la sociología de la ciencia tam- 
bién aparece como otra meta-disciplina que cierra junto a la historia y la filosofía de la ciencia un triángulo virtuoso que inaugura un programa que ha sido fértil y encontró en ERC su entrada triunfal (ver Hoyningen-Huene, 1993).

En la próxima sección nos referiremos a la importancia del programa historicista en filosofía de la ciencia y su impacto para una historia de la ciencia más fértil.

\section{Sobre la importancia del programa historicista en filosofía de la ciencia para la historia de la ciencia}

Antes de la inauguración del programa historicista en la filosofía de la ciencia existían dos perfiles (o tradiciones) típicos de investigador en historia de la ciencia, los cuales fueron descritos en detalle para la academia estadounidense de los 6os y 70 os por Kuhn (ver 1982b, 1982c y I982d).

El primer perfil refiere a aquellos cuya formación de grado es en historia, y se dedican a la historia de la ciencia como especialidad dentro de ésta. El segundo perfil refiere a aquellos que se forman como científicos de una especialidad (puede ser de las ciencias fácticas o formales) y luego se dedican a la historia de dicha especialidad.

El programa historicista en filosofía de la ciencia trajo a su vez un tercer perfil, el de personas formadas en filosofía de la ciencia que luego, tomando como base algún perfil epistemológico de preferencia han comenzado a indagar en el pasado, generando fértiles trabajos en historia de la ciencia.

No es descabellado pensar que estos perfiles aún permanezcan en la academia dado que las tradiciones de formación en historia o filosofía varían muy lentamente en el tiempo.

En su análisis de los perfiles I y 2, Kuhn observaba con preocupación ciertas características de los mismos que le restaban fertilidad a los productos de su investigación histórica. Los provenientes de la historia general, tendían a caracterizar a la ciencia como el triunfo de la razón sobre la superstición, y abundaban en notas eruditas sobre autores y fechas en desmedro de contenidos propiamente científicos. Por otro lado, los provenientes de una disciplina científica concreta (menciona entre éstos al emblemático George Sarton), tendían a realizar tratados que solían ser para Kuhn productos secundarios de una tarea pedagógica, y resultaban en gran medida anacrónicos, pecando de lo que David Hackett Fischer (1970) denominó la "falacia presentista", previamente 
caracterizada como "historia Whig" por Herbert Butterfield (I93I). Por supuesto, no podemos inducir desde dichas caracterizaciones que las mismas se repitan vís-a-vís para la academia actual pero tampoco podemos desestimar la posibildad. Está claro que las descripciones delineadas son producto de ciertas tendencias en la formación profesional de los historiadores, y no es ilógico considerar que el medio de evitar la cristalización de las mismas es mediante acciones concretas y conscientes de corrección de estas tendencias.

Buena parte de la obra de Kuhn está dedicada a formular análisis filosóficos e historiográficos cuyo fin es permitir a los historiadores realizar investigaciones más fértiles, dando lugar a una mejor comprensión de los procesos de progreso científico. Buena parte de estos textos fueron publicados originalmente en revistas de historia de la ciencia y no de filosofía. Decía Kuhn en la Conferencia Isenberg de 1968 "Me presento ante ustedes como historiador de la ciencia. Mis estudiantes, en su mayoría desean ser historiadores, no filósofos. Y yo soy miembro de la Asociación Norteamericana de Historia, no de filosofía" (I982b, p.27).

¿Qué puede aportar el perfil filosófico (perfil 3) a las investigaciones en historia de la ciencia?

Imre Lakatos, parafraseando a Kant afirmó que: "La filosofía de la ciencia sin la historia de la ciencia es vacía; la historia de la ciencia sin la filosofía de la ciencia es ciega" (1987, p. II). Así, la filosofía de la ciencia como disciplina tendría entre sus objetivos el de construir metodologías normativas para reconstruir episodios históricos, mientras la historia de la ciencia aportaría casuística con la que puede decidirse cuál entre dos metodologías es mejor.

Siguiendo a Lakatos, entonces, un historiador de la ciencia podría estudiar una determinada corriente epistemológica (por ejemplo, al inductivismo o al racionalismo popperiano) y capturar de sus lineamientos filosóficos un determinado método de trabajo a fin de poder reconstruir mediante dicho marco el episodio histórico de interés. Así, un historiador inductivista relatará su episodio histórico hablando de procesos de observación, generalización y contrastación, mientras un historiador lakatosiano describirá su episodio en términos de los elementos propios de un Programa de Investigación Científica que ha progresado o se ha degenerado después de una cantidad de procesos de heurística positiva o negativa.

Más allá de los compromisos filosóficos que lleven a un historiador a asimilar una u otra metodología normativa como la preferible, un filósofo puede tomar un episodio histórico determinado, reconstruirlo con 
dos metodologías normativas distintas y evaluar entonces las bondades de una u otra en base a ciertos objetivos historiográficos particulares.

Lakatos, sin embargo, toma la precaución de afirmar que estas metodologías normativas que se desprenden de marcos epistemológicos son " $[\mathrm{u}] \mathrm{n}$ conjunto de reglas (quizá no rigurosamente interrelacionadas, mucho menos mecánicas) para la evaluación de teorías ya elaboradas" y en nota al pie "El término "normativo" no tiene el significado amplio de reglas para llegar a soluciones, sino simplemente el de directrices para llegar a soluciones ya existentes" (p.I2). En otras palabras, las metodologías normativas no serían sino lineamientos generales, pistas para que el historiador interprete los episodios históricos mediante marcos coherentes a fin de poder evaluar su estructura, poder realizar comparaciones, etc.

De esta forma de considerar las cosas, parece poder derivarse incluso un criterio de demarcación entre la filosofía de la ciencia y la historia de la ciencia: la filosofía es "normativa" pues provee la metodología (normativa) para que el historiador haga su trabajo mediante un marco teórico bien explícito (y que haya sido discutido y analizado con rigor por parte de la comunidad... filosófica). Y por otra parte, la historia sería descriptiva, pues se limitaría a realizar relatos más o menos interesantes de los episodios históricos de la ciencia, valiéndose de las concepciones filosóficas y aportándoles a los normativistas casuística para la evaluación de dichas concepciones.

Esta distinción es cuestionada por Ulises Moulines (1986) al afirmar:

¿Qué gana el historiador en saber que los hechos que él investiga serán "postumamente" evaluados según algún criterio de buena ciencia en cuya evaluación él mismo no ha tenido nada que ver? ¿y de qué le sirve al filósofo normativo averiguar que un gran número de hechos históricos no cumplen con sus normas?

(...) Las normas que el filósofo formula con respecto a la ciencia deben estar subordinadas a una metanorma destinada a tranquilizar la conciencia del historiador: si el conjunto de reglas metodológicas propuestas por el filósofo conduce a una evaluación negativa de un gran número de hechos de la historia de la ciencia, entonces hay que revisar la metodología. Pero esto es hacer trampa. Es como si alguien recomendara que se abandonara cualquier código moral que contenga el principio "no matarás" por la razón de que un gran número de gente en el curso de la historia ha asesinado a sus congéneres. (pp. 56-57).

La aguda observación de Moulines nos recuerda que este juego en el que los filósofos proponen marcos y los historiadores los utilizan para "encorsetar" a sus episodios históricos no debe considerarse rígidamen- 
te o la consecuencia puede ser grave: la historia no entra perfectamente en ningún marco rígido (ver Nickles 1986), para frustración de filósofos e historiadores. Los marcos deben entonces ser flexibles para adaptarse a la historia tal como la describen las fuentes, lo cual no implica necesariamente la pérdida del rigor filosófico.

La renuncia al uso rígido de los marcos filosóficos no significa que el historiador no realice sus descripciones históricas a través del crisol de un marco teórico. La idea de una descripción histórica basada en la observación pura de las fuentes (escritas) propuesta por la escuela alemana capitaneada por Leopold von Ranke en el Siglo XIX es insostenible tras la aceptación de la presencia de carga teórica en la observación. Lo que propone la filosofía historicista es un uso flexible de los marcos, y sobre todo, su explicitación, que facilita la comprensión de los procesos históricos reseñados aumentando la fertilidad del análisis.

Sin un marco interpretativo, la historia no es más que un compilado de hechos sueltos.

Una narración histórica consiste principalmente en hechos acerca del pasado, la mayoría de ellos aparentemente indisputables. De ahí que muchos lectores supongan que la tarea primordial del historiador es la de examinar textos, extraer de ellos los hechos pertinentes, y examinarlos con gracia literaria, más o menos en orden cronológico. (Kuhn, I982a, p. Io).

Ya el propio Kuhn en ERC nos insta a considerar la historia "como algo más que un depósito de anécdotas o cronología..." para “...producir una transformación decisiva de la imagen que tenemos actualmente de la ciencia" (p. 20). Hacer historia será entonces no meramente el arte de describir hechos del pasado, sino el arte de describirlos bajo un marco interpretativo más o menos fértil de manera flexible. Claro está que en la comunidad de historiadores no hay un acuerdo completo en la actualidad respecto a la fertilidad del uso de marcos interpretativos filosóficos para la investigación en el área (ver, p.e., Daston 20I6), pero sobre todo desde la filosofía de la ciencia insistimos en la importancia de ofrecer a los historiadores esta valiosa herramienta de trabajo. Desgraciadamente, un análisis de los programas universitarios en historia en la región sudamericana arroja que la historia de la ciencia es marginal en la formación de historiadores, y, a su vez, la formación sobre la obra de los filósofos historicistas de la ciencia brilla por su ausencia, con la excepción del omnipresente ERC. Esperamos con este trabajo y con el avance en los estudios sobre Kuhn en general promover la introducción de estos temas en las currículas del área. 
En la próxima sección nos dedicaremos a recuperar el programa en historia de la ciencia de Kuhn. Nuestra hipótesis de trabajo es que de su obra puede destilarse un marco interpretativo para conformar un programa en historia de la ciencia, y que dicho marco ha progresado sustancialmente desde ERC en adelante, siendo DCCL el lugar donde es posible hallar con mayor sistematicidad la propuesta desde la letra del autor.

\section{Sobre el programa kuhniano en historia de la ciencia y sus debilidades}

En esta sección presentaremos esquemáticamente los lineamientos principales del marco interpretativo historiográfico kuhniano. Un análisis preciso sobre los elementos de los marcos historiográficos es el de Hoyningen-Huene (2012), quien sugiere que la historia de la ciencia precisa "criterios comparativos de relevancia histórica" para su práctica. Estos criterios permiten seleccionar los hechos a incluir en la narración histórica, aportando heurísticas para guiar la investigación. Tales criterios se pueden agrupar en tres tipos:

a) criterios de relevancia factual: selecciona el material relevante para que la historia de un determinado tópico científico pueda ser contada.

b) criterios de relevancia narrativa: selecciona el material para que la narración histórica tenga continuidad y fluidez.

c) criterios de relevancia pragmática: selecciona el material para lograr el objetivo histórico deseado, teniendo en cuenta el público target.

Dentro de tales criterios aparecen cuestiones filosóficamente relevantes, y por ello pueden ser llamados "elementos filosóficos del trabajo historiográfico". Incluirían

[l]os presupuestos usualmente implícitos acerca de la historia en sí misma, o acerca de la presentación e investigación histórica que influencia al trabajo histórico. Está claro que, por ejemplo, las decisiones acerca de los propósitos generales de la historiografía de la ciencia [...] o las convicciones acerca de la influencia de los factores sociales en el contenido de la ciencia cualifican tanto como criterios de relevancia histórica y como elementos filosóficos de la respectiva historiografía (p. 283). 
No debe sin embargo interpretarse que la filosofía provee criterios a modo de normas a priori, y que la historia deba recibirlos en forma pasiva para operar con ellos a posteriori. La interacción entre ambas disciplinas debe entenderse, como " $[\mathrm{u}] \mathrm{n}$ proceso de retroalimentación interactiva entre las preconcepciones de la ciencia y la investigación histórica concreta" (p. 286). Es en este proceso de feedback en que se puede dar que la historia de la ciencia aporte ingredientes correctivos a la filosofía de la ciencia, especialmente a la hora de sopesar los criterios narrativos, en donde los factores provistos por la filosofía de la ciencia son de menor peso que en los factuales o pragmáticos.

Un marco interpretativo bien construido contribuirá a evitar falacias típicas del trabajo historiográfico (ver Hackett Fischer, 1970). En particular, ERC se preocupa por la ya mencionada falacia presentista, bajo la cual

El desarrollo científico se convierte en el proceso gradual mediante el cual esos conceptos [los que actualmente aceptamos como válidos] han sido añadidos, solos y en combinación, al caudal creciente de la técnica y de los conocimientos científicos, y la historia de la ciencia se convierte en una disciplina que relata y registra esos incrementos sucesivos y los obstáculos que han inhibido su acumulación. Al interesarse por el desarrollo científico, el historiador parece entonces tener dos tareas principales. Por una parte, debe determinar por qué hombre y en qué momento fue descubierto o inventado cada hecho, ley o teoría científica contemporánea. Por otra, debe describir y explicar el conjunto de errores, mitos y supersticiones que impidieron una acumulación más rápida de los componentes del caudal científico. (Kuhn I97I, p.2I).

La historiografía presentista propone una cronología teleológica donde los actos relevantes del pasado son aquellos que sembraron la semilla para obtener el conocimiento científico actualmente válido, concibiéndose entonces dicho conocimiento como de naturaleza acumulativa. A su vez, el planteamiento de la dinámica científica implicaba que los descubrimientos eran más bien "momentos eureka" y no procesos de larga duración. Además, el método científico es considerado universal, y se juzga todo acontecimiento científico en términos de "bueno" o "malo" en relación al cuerpo de conocimiento aceptado actualmente. Así, los científicos son clasificados en ganadores (aquellos cuyas contribuciones permanecen hoy dentro de lo aceptado por la comunidad científica, vencedores en la guerra contra la metafísica irracional), y los perdedores (la mayoría de los cuales ni siquiera figurarán en las narraciones históricas, o lo harán en su carácter de vencidos). 
Hoyningen-Huene (2012) de hecho sugiere que el anti-presentismo es el principal criterio de relevancia histórica de toda la historiografia kuhniana, no solo de ERC. Entonces, cataloga a Kuhn como un historiador Anti-Whig, lo cual conlleva una metodología hermenéutica particular para abordar las fuentes, y un punto fuerte a tomar en cuenta para caracterizar el marco interpretativo kuhniano. Hoyningen-Huene considera que hay al menos tres elementos filosóficos constitutivos de la hermenéutica kuhniana: una hipótesis internalista, una racionalidad particular para la ciencia (que para Kuhn se relacionaría con ciertos valores epistémicos cuya valoración fluctúa en el tiempo y las comunidades pero en forma lenta y estable, ver Kuhn I982e) y una postura de realismo histórico (doctrina que implicaría que es posible conocer lo que realmente sucedió en la historia, no debe confundirse con posturas de realismo epistemológico con las que Kuhn no comulgaba, o dejó de comulgar luego de ERC).

Coincidiendo con Hoyningen-Huene, afirmamos que dichos elementos configuran la historiografía kuhniana en general. No profundizaremos en el problema de la racionalidad científica en Kuhn ni en su realismo histórico. Nos enfocaremos en tres puntos esenciales del marco historiográfico kuhniano y que han conllevado mucho trabajo de refinamiento por parte del autor a lo largo de su carrera. En primer lugar, los problemas del internalismo, el cual, planteado del modo en que lo fue en ERC llevó a la formación de una escuela supuestamente kuhniana pero profundamente divergente de las convicciones de Kuhn, de carácter metafísico relativista. En segundo lugar, nos concentraremos en los problemas que conlleva el anti-presentismo en la historiografía.

Además, profundizaremos en un tercer elemento fundamental: la propuesta de dinámica científica por paradigmas presentada por Kuhn en ERC. Dicha propuesta describe la génesis de las disciplinas de las ciencias naturales y su evolución. Así, si se pretende hacer historia de la ciencia kuhniana, una parte del trabajo será ubicar a la teoría investigada dentro de alguna de las fases sugeridas por Kuhn, de modo de poder establecer comparaciones y preguntas respecto a la ocurrencia o no de lo esperado de acuerdo al esquema dinámico. En definitiva, la dinámica de la ciencia de Kuhn constituye una de las referencias para el historiador a la hora de desempeñar su trabajo hermenéutico, razón por la cual será una de las dimensiones a analizar si se quiere entender la constitución del marco interpretativo kuhniano. Por ser la dimensión más conocida del trabajo kuhniano seremos sintéticos con el análisis de la propuesta en esta sección, dejando para la próxima los cambios en su abordaje en DCCL. 
a) Sobre el internalismo kuhniano (y su relativismo asociado)

Mladenovic (2007) afirma que el internalismo desarrollado por Kuhn en ERC tuvo una consecuencia imprevista y nefasta para la concepción del autor: el dar soporte teórico al origen del Programa Fuerte de Edimburgo en Sociología de la Ciencia y con él a su escuela historiográfica derivada (p.e., Barnes I982). Este hecho llevó a que buena parte del trabajo posterior de Kuhn se dedicase a refinar su posición metafísica de modo tal que el relativismo pregonado por el Programa Fuerte no formase parte de su propuesta filosófica e historiográfica.

La tesis central del Programa Fuerte era el "principio de simetría" que sostenía que debía utilizarse el mismo tipo de explicación para las teorías científicas satisfactorias que para las insatisfactorias (ver Barnes \& Bloor 1982). Esto era una respuesta a la concepción tradicional que consideraba a las teorías satisfactorias como fruto de la aplicación correcta del método científico, mientras se volvía externalista para las insatisfactorias, derivando en explicaciones sociológicas para éstas. Dicha concepción se denominó "sociología del error" (ver, p.e., Lynch \& Woolgar I988), heredera de la distinción neopositivista entre "contexto de descubrimiento" y "contexto de justificación” (Reichenbach I96I). Entonces, los sociólogos del Programa Fuerte se plantearon aportar explicaciones causales para la adopción de teorías, explicaciones de orden casi siempre psicológicas, sociales o políticas, tanto para las aceptadas como para las rechazadas.

La historiografía derivada del trabajo del Programa Fuerte contempló entonces explicaciones socio-políticas para el desarrollo de ciertos descubrimientos científicos, en desmedro de las cuestiones internas a las propias ciencias interesadas (un ejemplo paradigmático es Shapin y Schaffer 1985). Este relativismo, si bien se autodenominó kuhniano, se encuentra en las antípodas de la filosofía y la historiografía kuhnianas.

Kuhn (2002a) detalla los puntos en que el Programa Fuerte y sus sucesores tergiversaron los preceptos con los que construyó su historiografía anti-presentista. Mladenovic (2007) plantea que

Los modelos explicativos del desarrollo científico están subdeterminados por los principios de la historiografía interna, y por ende estos principios no pueden ser de ayuda contra modelos explicativos rivales, igualmente firmes hermenéuticamente. [la metodología hermenéutica kuhniana en ERC] era incapaz de proveer principios resistentes contra las categorías explicativas sociológicas a expensas de las cognitivas [las que Kuhn defendía], y quedaba indefensa contra el relativismo resultante. [...] es explícitamente 
pluralista al permitir diferentes explicaciones legítimas del mismo fenómeno empírico, motivadas por diferentes intereses explicativos (p. 285).

Coincidimos con la autora al afirmar que los de Edimburgo no tergiversaron ERC, sino que tomaron uno de los caminos que el opus magnum de Kuhn dejó habilitados. ¿Cómo?

[a]l insistir que ni la lógica ni la evidencia empírica determina la elección entre hipótesis reales o paradigmas, Kuhn pensó que estaba sólo haciendo lugar para la comprensión de los desacuerdos científicos del pasado como desacuerdos entre personas racionales que interpretaban la evidencia legítimamente y aplicaban los valores científicos de maneras diferentes. [...] La escuela sociológica argumentó que, en ausencia de evidencia convincente, la elección científica debe ser explicada como determinada por idiosincrasias personales, ambiciones e intereses. Esto implica que la elección que fue eventualmente realizada no podría haber sido tratada en ningún sentido cognitivamente mejor por su rival histórico. La historia de las ideas en general, y la historia de la ciencia en particular, se volvió, bajo el modelo explicativo de la escuela sociológica, esencialmente similar a las historias de lucha política por la dominancia. (Mladenovic 2007, pp. 285-286).

Así es como la propuesta internalista de ERC dio origen a la escuela sociológica, de corte externalista. Kuhn afirmó que

los autores de los estudios microsociológicos se exceden en sus concesiones al punto de vista tradicional del conocimiento científico. Es decir, parecen creer que la filosofía de la ciencia tradicional era correcta en su comprensión de lo que el conocimiento debe ser. Lo primero deben ser los hechos, y las conclusiones inevitables, al menos sobre las probabilidades, deben basarse en ellos. Si la ciencia no produce conocimiento en este sentido, concluyen, no puede producir conocimiento en absoluto. (2002b, pp. 137-I38).

Así, la escuela sociológica plantea que como la epistemología kuhniana sostiene correctamente que esta concepción de "hecho" tradicional (objetiva e incuestionable) es insostenible dada la carga teórica de la observación, pues entonces no hay conocimiento científico derivable del mundo, y todo lo que se dice tendría su origen en las "negociaciones" entre agentes, negociaciones que se hallan moldeadas por factores de autoridad y poder. Esta escuela también tomó de Kuhn su anti-presentismo, aunque lo llevó a un extremo inaceptable para el autor. Según Hoyningen-Huene (20I2), la influencia de los de Edimburgo llevó a que se etiquete peyorativamente como "Whig" a cualquier postura historiográfica que presuponga apenas que la ciencia progresa de alguna forma 
o que pretenda abrir juicios en términos de "correcto o incorrecto" sobre las acciones de los actores históricos. Kuhn en este sentido sería presentista, pues acepta el progreso científico, cosa que permite ver otro motivo de disconformidad del autor. Aclarar estas cuestiones llevó muchas páginas de esfuerzo para Kuhn.

\section{b) Sobre el anti-presentismo de Kuhn}

Hemos visto que el Programa Fuerte puso en tensión las categorías de presentismo/anti-presentismo al exacerbar el repudio al whiggismo. Kuhn se posicionó como Anti-Whig pero distanciándose de los sociólogos. Cabe afirmar sin embargo que toda historiografía Anti-Whig posee una serie de imposibilidades esenciales que un defensor de esta visión debe explicar cómo puede superar sin traicionar su posición:

a. Comunicar a una audiencia actual los eventos científicos del pasado, pues considera etnocentrista utilizar el lenguaje actual: la ciencia contemporánea resulta inevitable al menos como contraste de la antigua a la hora de comunicar, pero esto resulta contrario al ethos Anti-Whig.

b. Comunicar situaciones que eran problemáticas para los científicos del pasado pero resultan diáfanas a la luz de la ciencia actual: nuevamente, hablar del pasado haciendo referencia a lo que se (cree que) se sabe en el presente es inevitable pero ideológicamente chocante para el Anti-Whiggismo.

c. Realizar preguntas legítimas del trabajo historiográfico, pero catalogables como Whig. No debe confundirse las preguntas con las respuestas, ya que es en estas últimas que el Whiggismo es peligroso. Por ejemplo: “ipor qué una teoría x que hoy aceptamos sin problemas tardó tanto tiempo en ser adoptada por la comunidad de su época?" (ver Hoyningen-Huene 2012).

Kuhn también ha tenido que enfrentarse a estos problemas luego de declarar su anti-presentismo en ERC. Veremos en la próxima sección la respuesta del autor a estos interrogantes.

c) Sobre la dinámica científica en ERC

Uno de los puntos por los que ERC es más recordado es por la propuesta de dinámica científica que provee una síntesis dinámica a de los marcos 
inductivista y popperiano. En ella la ciencia avanza desde un período inmaduro o preparadigmático hacia la formación de un paradigma, el cual pasa por las etapas de ciencia normal (de resolución de puzzles), crisis (por acumulación de anomalías) y revolución, al sustituir un paradigma por otro capaz de lidiar con las anomalías irresueltas por su predecesor. Esta propuesta ha demostrado ser exitosa en múltiples áreas del saber, volviendo el concepto de paradigma ubicuo en el vocabulario académico. Sin embargo, las críticas a la ambigüedad del concepto (ver Masterman 1970) llevaron a Kuhn a desambiguar en la Posdata del 69 a ERC al término "paradigma" en "matriz disciplinar" y "ejemplar" (el primero como unidad de análisis de la ciencia y el segundo como caso exitoso de resolución de problemas dentro de la matriz disciplinar). Además, varios filósofos (p.e., Popper 1970; Watkins 1970) se ocuparon con fruición de atacar la propuesta dinámica de Kuhn, pues la resistencia de la ciencia normal en eliminar los enunciados que no aprueban los tests empíricos contradice la esencia del ethos crítico por ellos defendido. Si bien Kuhn se ha defendido de tales críticas (p.e., Kuhn 1970), lo cierto es que la propuesta de dinámica de ERC no es retomada explícitamente en los libros posteriores de Kuhn, y ha mutado su forma e incluso su unidad de análisis. Esta renuncia parcial a continuar desarrollando esta propuesta debe entenderse como crucial a la hora de abordar la hermenéutica kuhniana y a su marco interpretativo historiográfico.

Todo este análisis apunta a mostrar que ERC, a pesar de su éxito perenne, dadas sus limitaciones inauguró un programa de investigación que ocuparía a Kuhn por el resto de su vida, programa que consistiría en salvar las problemáticas suscitadas por su metateoría. Como mencionamos, una versión sistemática y coherente del producto de tal programa se encuentra en DCCL. En lo que queda de este trabajo analizaremos las propuestas que Kuhn plantea en esta obra para apuntalar las problemáticas que debilitaban su programa historiográfico.

\section{El programa historiográfico en DCCL}

En lo que sigue del artículo describiremos en forma sintética aquellos elementos de DCCL con los que Kuhn busca salvar las problemáticas acarreadas por su posición historiográfica en ERC, presentadas en la sección anterior. De este modo fundamentaremos por qué creemos que este texto es un buen candidato para ser utilizado como medio pedagógicamente accesible de presentar la posición kuhniana. Las problemáticas a tratar serán entonces: 
a. Asentamiento de las bases filosóficas para el surgimiento de la escuela sociológica (y habilitación de críticas desde la filosofía de la ciencia por sostener una supuesta versión irracional de la elección de teorías).

b. Fundación de una historiografía Anti-Whig, y por ende teñida por sus virtudes y limitaciones.

c. Invocamiento de cuestionamientos filosóficos a su modelo de dinámica científica a través de paradigmas.

Veremos a continuación las respuestas que se le dan a estos tópicos problemáticos del marco historiográfico kuhniano en DCCL.

\section{a) Respuestas al problema del relativismo}

Ya hemos visto que una de las críticas más fuertes que se han esbozado contra ERC es acerca de su posición "relativista": a menudo se ha interpretado a Kuhn defendiendo la idea de que un cambio de paradigma arrastra un cambio de mundo, y luego, el mundo es relativo a la comunidad, y las elecciones de teorías son por ende irracionales. Si no hay correspondencia entre una teoría científica y un mundo independiente de la conciencia humana, los cambios de teorías serían en base a factores extracientíficos. Kuhn necesitó refinar la posición metafísica para contestarle a los epistemólogos que criticaron el supuesto relativismo kuhniano como también a los sociólogos que la recibieron de brazos abiertos, ambos persiguiendo una hipótesis explicativa del cambio teórico a la que el autor no adhería. Menciona Melogno (2017) que

Por un lado, "relativo" puede ser entendido como "dependiente de", "subordinado a" y por otro como "arbitrario" o "carente de fundamento". El primer sentido implica sólo una relación de dependencia entre factores [...], mientras que el segundo se asocia frecuentemente a la idea de que nociones como "verdad", "racionalidad" y "progreso", son relativas a cada sujeto, comunidad o contexto, de forma que lo que se diga en torno a ellas responde a convenciones más o menos arbitrarias, antes que a argumentos lógicos o pruebas empíricas (pp. 38-39).

Las acusaciones contra Kuhn (y que son constitutivas al Programa Fuerte) corresponden a este segundo sentido de "relativismo". Pero la inconmensurabilidad kuhniana post-ERC no impide la comparación de léxicos sucesivos: no puede entonces atribuírsele a Kuhn este sentido de relativismo. El relativismo kuhniano es de carácter lingüístico, y consiste 
en la no-intertraducibilidad de dos lenguajes científicos sucesivos que lleva a asignaciones variantes del valor de verdad de ciertos enunciados (ver, p.e., Kitcher 1982).

Este refinamiento es menester para resolver la acusación de irracionalidad por un lado y el malestar que produce la interpretación de la escuela sociológica por el otro. Kuhn no piensa que las decisiones que toman las comunidades científicas no tengan relación alguna con aquello que hay en el mundo, puesto que dicho mundo posee relación causal con lo que perciben las personas. Existe entonces una suerte de plataforma arquimedeana para la verificación de teorías, solo que no sería fija e independiente del contexto como hubieran querido Bacon y Descartes, sino que es móvil y muta lentamente acompañando la evolución del lenguaje científico.

¿Es esta una posición idealista? Quizás lo sea. Pero entonces este idealismo es distinto a todos los otros de los cuales tengo noticia. Las ideas proyectadas hacia afuera para hacer un mundo no son las de un individuo, ya sea humano o divino, sino más bien las de la tribu. Y esto implica decir que no son propiamente ideas sino lazos compartidos, que constituyen una comunidad que ha sobrevivido por largo tiempo en un mundo, y que han desempeñado un rol en la constitución de dicho mundo a lo largo de la historia. Ese mundo está poblado por los referentes de términos que pueden ser transmitidos por ostensión de una generación a la siguiente, señalando ejemplos que hay allí fuera, en el mundo. $Y$ es un mundo con solidez suficiente para refutar a aquellos que lo torcerían para adecuarlo a sus intereses o voluntades individuales. Quizás es con todo el mundo de un idealista, pero se siente muy real para mí. (Kuhn 20I7, p.I48, énfasis añadido)

Este tipo de relativismo es completamente distinto al del Programa Fuerte, por lo que el posicionamiento de Kuhn despeja toda duda respecto a la compatibilidad de ambos programas. Las decisiones de grupo no pueden doblegar al mundo, por lo que la clave para la historia de la ciencia está en la ciencia misma y no en el grupo. El marco historiográfico, si es kuhniano, no puede ser radicalmente externalista.

b) Respuestas a los problemas del anti-presentismo:

Sobre los límites de la historiografía Anti-Whig, es Davidson en 1974 quien cuestiona la posibilidad de reconstruir con el lenguaje actual las problemáticas científicas antiguas: si los lenguajes son realmente inconmensurables (y por ende intraducibles) no sería posible el trabajo historiográfico, y mucho menos desde la óptica anti-presentista que pretende 
etnocentrista y falaz cualquier intento de descripción de lo antiguo con el lenguaje moderno (ver Davidson 1990).

Kuhn dedicará un gran esfuerzo en DCCL a esta problemática que considera fundamental. En primer lugar, el autor diferenciará entre "comprender" y "traducir": la imposibilidad de un manual de traducción quineano entre dos lenguajes inconmensurables no implica que un historiador no pueda comprender en su trabajo hermenéutico el lenguaje del pasado. Es el propio trabajo hermenéutico el que habilita la comprensión sin traducción. Pero este trabajo requiere una tenaz investigación sobre el campo lingüístico (será fundamental ampararse en la base semántica común de los dos léxicos, ya que fuera de ERC Kuhn aclaró que la inconmensurabilidad no debe ser considerada total sino parcial, y la parte compartida es la que habilita las comparaciones), los factores contextuales que motivaron su constitución, los problemas que interesaban a la comunidad antigua, las prácticas y las instituciones de la época analizada.

Una narrativa histórica debe proveer a sus lectores un enfoque plausible del desarrollo. Los eventos que registra pueden ser de un tipo no familiar para ellos, pero tanto los eventos como su sucesión deben tener sentido. El historiador debe, en pocas palabras, eliminar las anomalías recurrentes que invariablemente resultan de los intentos de ajustar el pasado al léxico del presente. Una narrativa histórica libre de anomalías solo puede ser escrita en el lenguaje del tiempo que describe. Si el tiempo cubierto por la narrativa es largo, entonces puede requerirse una serie completa de léxicos.

Afortunadamente, proveer una narrativa de este tipo no es tan difícil como puede parecer. Los historiadores lo han estado haciendo por mucho tiempo, si bien no siempre han sido lo suficientemente autoconscientes como para hacerlo bien. La mayoría de los libros de historia comienzan preparando el escenario, describiendo la cultura de los actores sobre los cuales el telón de apertura se elevará, lo que creían acerca del mundo y de las criaturas en él, qué instituciones dieron forma a sus vidas y a las de sus contemporáneos. Si bien tales preliminares históricos no son anunciadas como enseñanza del lenguaje, ellas, inter alia, proveen a los lectores el léxico que necesitarán cuando la lectura avance. Especifican los referentes de los términos en el léxico de los actores históricos, describen las características principales de aquellos referentes, y dicen algo acerca de la manera en que se interrelacionan. (Kuhn 20I7, pp. I34-I35).

Como actitud metodológica, agrega Kuhn, se hace necesario una suerte de "caridad argumentativa": el historiador debe considerar que el científico antiguo es un individuo inteligente y por ende no partir de 
la base de que aquellos postulados que hoy consideramos perimidos eran erróneos, sino que en el mundo de aquel científico (con su lenguaje y su plataforma arquimedeana de contrastación) podrían haber sido correctos.

Primero, él o ella debe descubrir y explicar cómo estas antiguas doctrinas (a menudo las más extrañas y aparentemente implausibles) pudieron haber sido aceptadas por gente inteligente como base para una tradición de larga data de la práctica científica (Kuhn 2017, p. 6I).

Este trabajo permite comprender al científico antiguo y su lenguaje, luego de lo cual puede comunicarse a una audiencia actual, sin pecar del típico etnocentrismo Whig.

Segundo, el historiador, debe buscar entender cómo y por qué el estatus de aquellas creencias cambió, qué llevó a su desplazamiento por otro conjunto y cómo las fronteras de la investigación fueron modificadas a consecuencia del cambio. Brevemente, para el historiador, a diferencia del filósofo tradicional de la ciencia, el avance de la ciencia está marcado menos por la conquista de la ignorancia que por la transición desde un cuerpo de afirmaciones de conocimiento a otro diferente, aunque solapado con el primero. Una explicación de dicha transición por lo tanto debe primero exhibir la integridad del antiguo cuerpo, y luego examinar su desplazamiento (Kuhn 20I7, p. 6I).

De todas formas hay que tener en cuenta que "[u]n enfoque diacrónico estricto es utópico. Nadie escapa a los "anteojos del presente" que le permiten acceder a una mirada histórica" (Boido 1993, p.I7). Si se trabajara únicamente con el lenguaje antiguo y con preguntas válidas acerca del contexto antiguo, la historia de la ciencia sería solo para unos pocos especialistas. Así, hemos de concluir que "[p]ara el historiador actual las visiones Whig y Anti-Whig no son excluyentes, y el conflicto desaparece una vez determinada la naturaleza del problema histórico en estudio" (Boido 1993, p. 2I). A esto refiere Hoyningen-Huene (2012) al referir sobre la legitimidad de ciertas preguntas Whig. No toda pregunta presentista es incompatible con el marco kuhniano sino que el autor discute contra aquellos que afirman

"Lo que me importa es lo que Aristóteles (o Galileo, o Volta) pensaban acerca de lo que nosotros llamamos movimiento (o fuerza, o resistencia)". Estas preguntas están mal formuladas: ni los historiadores ni nadie más puede responderlas. Posiblemente pueden surgir de una necesidad humana fundamental pero que no puede ser satisfecha. (Kuhn 20I7, p. I36). 
c) Sobre la dinámica científica (y cómo es tratada después de ERC).

Bird (20I5) afirma que así como la idea de "ciencia normal" era contraria a la visión popperiana ya que este creía que los científicos debían rechazar las teorías falsadas, los inductivistas encontraban en dicha conceptualización una etapa compatible con su concepción acumulativa del cambio científico. Era la ciencia extraordinaria la problemática en este caso, ya que el rupturismo de tal etapa da por tierra el perenne progreso lineal del conocimiento científico. Más allá de que Kuhn defendió su dinámica cíclica ante las protestas de la comunidad filosófica, lo cierto es que para el momento en que pronuncia las Conferencias Thalheimer, la forma en que analiza el progreso científico ya no utiliza al "paradigma" (o matriz disciplinar) como unidad epistemológica de análisis. El progreso científico será entonces tratado como un proceso en el que simplemente un "cuerpo de conocimiento" es desplazado por otro. Kuhn no profundiza en lo que entiende por "cuerpo de conocimiento", pero sí da la pauta de que estaría conformado por el conocimiento de la naturaleza compartido por la comunidad/tribu y el léxico que utiliza para expresar sus enunciados al respecto. Se trataría entonces de una estructura conformada por las entidades que pueblan el mundo y por los términos que utiliza la comunidad para nombrarlas. En definitiva, Kuhn no abandonó su modelo del cambio científico, sino que dedicó más esfuerzos a estudiar el cambio lexical como locus de la dinámica científica.

Kuhn ha cambiado en forma sustantiva el aumento de su lente historiográfica, pues su nueva unidad de análisis es el "término", nodo de la red lexical que conforma el mundo en el que viven y trabajan los científicos.

Puede notarse que si bien en esencia el pensamiento kuhniano no ha variado de manera sustancial desde los tiempos de ERC hacia su etapa más refinada, sí han cambiado las prioridades analíticas y ha surgido la necesidad de profundizar varias nociones, y por ello el marco interpretativo se ha modificado drásticamente. Los historiadores que pretendan trabajar bajo este marco ya no intentarán mostrar la presencia de los típicos elementos de un paradigma en el episodio analizado, sino que concentrarán su arsenal analítico en la interpretación del léxico antiguo y la reconstrucción del mundo de la época a partir de él. En DCCL la clave de la narración histórica está en el análisis de los mapas lexicales que conectan a los términos con sus entes asociados, y por ende sería provechoso enseñar a los futuros historiadores esta cara del autor que hasta su publicación parecía reservada sólo para los epistemólogos. Es 
muy probable que obtengamos trabajos historiográficos más fértiles, o al menos más cercanos a lo que Kuhn nos quiso legar.

\section{Conclusiones}

El texto de Kuhn que más relevancia tuvo en la historia y la filosofía fue ERC, el cual aún a más de 50 años de su publicación original sigue siendo la piedra basal de los estudios de la obra del autor. Más tarde fue refinando la posición allí presentada mediante la consideración de algunas de las críticas recibidas. En 1984, durante las Conferencias Thalheimer en la Universidad Johns Hopkins, el autor presentó una condensación sistemática de muchos de los conceptos utilizados en su libro más popular y sus correcciones, aunque tal conferencia no ha sido publicada hasta más de 30 años después y en idioma español, en DCCL. Esto configura una oportunidad única para utilizar una mejor versión de la obra de Kuhn para su uso en investigación y enseñanza. Si bien, lo antedicho es válido para los aspectos filosóficos e historiográficos, aquí nos hemos ocupado de estos últimos, pues hemos afirmado que DCCL constituye un texto de importancia capital para reconstruir el marco historiográfico kuhniano desde una fuente primaria sistemática.

Aquí nos hemos ocupado de tres aspectos del marco interpretativo historiográfico de Kuhn que fueron registrados en su forma refinada en DCCL. En primer lugar, Kuhn refinó su posición internalista para no dar lugar a la idea de que las elecciones de teorías son irracionales, aclarando que los cambios de teorías no son netamente en base a factores extracientíficos. En segundo lugar, Kuhn refinó su anti-presentismo a fin de sostener la posibilidad conceptual de progreso científico racional al contemplar una metodología hermenéutica por la cual un hablante de un lenguaje científico es capaz de entender al hablante de un lenguaje antecesor. En tercer lugar, Kuhn modificó su comprensión de la dinámica científica: el "paradigma" (o matriz disciplinar) dejó de ser la unidad de análisis fundamental de su método historiográfico, para poner el foco sobre el cambio lexical. En DCCL Kuhn sostiene que se entiende por progreso científico al desplazamiento de un cuerpo de conocimiento por otro, conformado por las creencias de una comunidad/tribu y el léxico que utiliza para expresar sus enunciados, por lo que remarca que es fundamental estudiar los nodos lexicales que conforman el mundo en que viven y trabajan los científicos.

Estas cuestiones son conocidas por los eruditos de Kuhn a través del análisis de los textos post-ERC o de las múltiples exégesis de su obra, 
pero quedan fuera del alcance de los estudiantes de historia que, como usualmente ocurre, son introducidos de manera superficial a la historiografía kuhniana únicamente a través de ERC.

Los tópicos analizados resultan de vital importancia para tenerlas en cuenta a la hora de realizar una historia de la ciencia tal como Kuhn la concibió. Son las bases del marco interpretativo historiográfico del Kuhn más maduro, recogiendo sus progresos sustanciales tras 20 años de trabajo post-ERC. La publicación de DCCL resulta invaluable en este sentido.

\section{Referencias bibliográficas}

Bar-Am, N. (20I4). "The Structure of Scientific Revolutions: 5oth Anniversary Edition: Review Essay". Philosophy of the Social Sciences. 44(5): 688-70I.

Barnes, B. (1982). T. S. Kuhn and Social Science. London: MacMillan Press. Barnes, B. y Bloor, D. (1982). "Relativism, Rationalism and the Sociology of Knowledge”. En M. Hollis y S. Lukes (eds.) Rationality and Relativism, 2I-47. Cambridge: MIT Press.

Bird, A. (2015). "Kuhn and the Historiography of Science". En A. Bokulich y W. Devlin (eds.) Kuhn's Structure of Scientific Revolutions - 50 Years On, I-I6. Cham: Springer.

Boido, G. (1993). "La polémica sobre el enfoque Whig en la historia de la ciencia". Análisis Filosófico, XIII(2): 13-22.

Butterfield, H. (193I). The Whig Interpretation of History. London: Norton. Daston, L. (2016). "History of Science Without Structure". En R. Richards y L. Daston (eds.) Kuhn's Structure of Scientific Revolutions at Fifty: Reflections on a Science Classic, II5-I32. Chicago: University of Chicago Press.

Davidson, D. (1990). "De la idea misma de un esquema conceptual". En D. Davidson, De la verdad y de la interpretación, I89-203. Barcelona: Gedisa.

Green, E. (2016). What are the Most-Cited Publications in the Social Sciences (According to Google Scholar)?. Disponible en http:// blogs.lse.ac.uk/impactofsocialsciences/2016/05/12/what-are-themost-cited-publications-in-the-social-sciences-according-togoogle-scholar/ [Consulta: 29/06/2019].

Hackett Fischer, D. (1970). Historians' Fallacies: Toward a Logic of Historical Thought. New York: Harper \& Row. 
Hacking, I. (2012), "Introductory Essay". En T. Kuhn, The Structure of Scientific Revolutions: 5oth Anniversary Edition, 6-63. Chicago: University of Chicago Press.

Hoyningen-Huene, P. (1993). Reconstructing Scientific Revolutions: Thomas S. Kuhn's Philosophy of Science. Chicago: Chicago University Press.

- (2012). "Philosophical Elements in Thomas Kuhn's Historiography of Science". Theoria, 75: 28I-292.

Kitcher, P. (1982). "Implications of Incommensurability". En Proceedings of the Biennial Meeting of the Philosophy of Science Association, Vol. 2, 689-703. Chicago: University of Chicago Press.

Kuhn, T. (1970). "Reflections on my critics". En I. Lakatos y A. Musgrave (eds.) Criticism and the Growth of Knowledge, 23I-278. London: Cambridge University Press.

(I97I [1962]). La estructura de las revoluciones científicas. México: F.C.E.

(I982a). "Prefacio". En T. Kuhn, La tensión esencial, I-25. México: F.C.E.

(1982b). "Las relaciones entre la historia y la filosofía de la ciencia”. En T. Kuhn, La tensión esencial, 27-45. México: F.C.E.

(I982C). "La historia de la ciencia". En T. Kuhn, La tensión esencial, I29-I50. México: F.C.E.

(1982d). "Las relaciones entre la historia y la historia de la ciencia”. En T. Kuhn, La tensión esencial, I5I-I85. México: F.C.E.

(I982e). "Objetividad, juicios de valor y elección de teoría". En T. Kuhn, La tensión esencial, 344-364. México: F.C.E.

(2002a). "Epílogo". En J. Conant y J. Haugeland (comps.) El camino desde la estructura, 267-298. Barcelona: Paidós.

(2002b). "El problema con la filosofía de la ciencia histórica".

En J. Conant y J. Haugeland (comps.) El camino desde la estructura, I3I-I48. Barcelona: Paidós.

(2017). Desarrollo científico y cambio de léxico. Montevideo: FICUDELAR, ANII, SADAF.

Lakatos, I. (1987). "La historia de la ciencia y sus reconstrucciones racionales". En I. Lakatos (ed.). La historia de la ciencia y sus reconstrucciones racionales, 9-78. Madrid: Tecnos.

Lynch, M y Woolgar, S. (1988). "Introduction: Sociological Orientations to Representational Practice in Science". Human Studies, II(2-3): 99-II6.

Masterman, M. (1970). "The Nature of a Paradigm". En I. Lakatos y A. Musgrave (eds.) Criticism and the Growth of Knowledge, 59-90. London: Cambridge University Press. 
Melogno, P. (2017). "Retornando al pasado". En T. Kuhn, Desarrollo científico y cambio de léxico, II-45. Montevideo: FIC-UDELAR, ANII, SADAF.

Mladenovic, B. (2007). “"Muckracking in History”: the Role of the History of Science in Kuhn's Philosophy". Perspectives on Science, 15(3): 26I294.

Moulines, U. (1986). "Filosofía de la ciencia - Historiografía de la ciencia: ¿dos caras de la misma medalla?". En Actas del III Congreso de la Sociedad Española de Historia de las Ciencias: San Sebastián, I al 6 de octubre de 1984, 53-66. San Sebastián: Sociedad Española de Historia de las Ciencias y de las Técnicas.

Nickles, T. (1986). "Remarks on the use of History as Evidence". Synthese, 69(2): 253-266.

Popper, K. (1970). "Normal Science and Its Dangers". En I. Lakatos y A. Musgrave (eds.) Criticism and the Growth of Knowledge, 5I-58. London: Cambridge University Press.

Reichenbach, H. (196I). Experience and Prediction. Chicago: Chicago University Press.

Shapere, D. (1964). "The Structure of Scientific Revolutions". Philosophical Review, 73: 383-394.

Shapin, S. y Schaffer, S. (1985). Leviathan and the Air Pump: Hobbes, Boyle and the Experimental Life. Princeton: Princeton University Press.

Scheffler, I. (1967). Science and Subjectivity. Indianapolis: Bobbs-Merrill.

Watkins, J. (1970). "Against 'Normal Science'". En I. Lakatos y A. Musgrave (eds.) Criticism and the Growth of Knowledge, 25-38. London: Cambridge University Press. 